\title{
Long Noncoding RNA snaR Regulates Proliferation, Migration and Invasion of Triple-negative Breast Cancer Cells
}

\author{
JEEYEON LEE ${ }^{1}$, JIN HYANG JUNG ${ }^{1}$, YEE SOO CHAE ${ }^{2}$, HO YONG PARK ${ }^{1}$, WAN WOOK KIM ${ }^{1}$, \\ SOO JUNG LEE ${ }^{2}$, JAE-HWAN JEONG ${ }^{3}$ and SEUNG HEE KANG ${ }^{3}$ \\ Departments of ${ }^{1}$ Surgery and ${ }^{2}$ Hemato-Oncology, ${ }^{3}$ Cell and Matrix Research Institute, \\ Kyungpook National University School of Medicine, Daegu, Republic of Korea
}

\begin{abstract}
Aim: We evaluated the role of long noncoding ribonucleic acid (IncRNA) in breast cancer cell lines by quantitative reverse transcription-polymerase change reaction. Materials and Methods: The effects of small NF90-associated RNA (snaR) with RNA interference on proliferation, migration and invasion of MDA-MB-231 cells were observed by 3-(4,5dimethylthiazol-2yl)-2,5-diphenyltetrazolium bromide, wound healing and transwell assay. Results: Among 90 lncRNAs, E2F transcription factor 4, p107/p130-binding (E2F4) antisense, insulin-like growth factor 2 antisense (IGF2AS), snaR, and small nucleolar RNA host gene 5 (SNHG5) were up-regulated in MDA-MB-231 and 7SK, antisense noncoding RNA in the INK4 locus (ANRIL), IGF2AS, Nespas, p53 mRNA, and snaR were up-regulated in MCF-7 cells. Down-regulation of snaR inhibited the proliferation, migration, and invasion of MDAMD-231 breast cancer cells. Conclusion: LncRNA snaR was found to be up-regulated in breast cancer cells, and the cancer progression of $M D A-M B-231$ cells was significantly suppressed by down-regulation of snaR. Therefore, snaR knockdown has potential as a treatment modality for triplenegative breast cancer.
\end{abstract}

Different types of noncoding RNAs have been identified and revealed to regulate the expression of various target genes. In particular, small noncoding RNAs, such as small interfering RNAs (siRNAs), microRNAs (miRNAs) and Piwi-interacting RNAs (piRNAs), have been extensively investigated, and

*These Authors contributed equally to this work.

Correspondence to: Jin Hyang Jung and Yee Soo Chae, Breast Cancer Center, Kyungpook National University School of Medicine, Hoguk-ro 807, Buk-gu, Daegu 4140, Republic of Korea. Tel: +82 532002702, Fax: +82 532002072, e-mail: jjh01@knu.ac.kr and yschae@knu.ac.kr

Key Words: Long noncoding RNA, breast cancer, triple-negative, progression. their underlying molecular mechanisms have been well documented in transcriptional and post-transcriptional processes. Long noncoding RNAs (lncRNAs), a new class of transcript, were recently revealed to be widely transcribed in human and mouse genomes; these are non-protein-coding transcripts longer than 200 nucleotides. However, the detailed biological function and mechanism of action of most lncRNAs are still being identified (1). Based on previous studies, lncRNAs may participate in a diverse range of biological processes from cell-cycle control to cell differentiation through distinct mechanisms at the transcriptional, post-transcriptional, and epigenetic regulatory levels, suggesting that lncRNAs serve as oncogenic or tumorsuppressor genes similar to protein-coding genes $(2,3)$.

It has been shown that lncRNAs play a major role as geneexpression regulatory factors which modulate tumorigenesis or promote tumor inhibition $(1,4,5)$. Although no specific drug against lncRNAs has been developed, the modulation of IncRNAs associated with a specific cancer type may be a novel therapeutic target. In particular, triple-negative breast cancer (TNBC), which is characterized by the lack of hormone receptor and human epidermal growth factor receptor 2 (HER2) gene expression, is difficult to treat by any immunomodulating agent except chemotherapy.

In the current study, we explored the expression of lncRNAs in various breast cancer cell lines based on the pathological subtypes and further investigated the role of small NF90-associated RNA (snaR) in proliferation, migration, and invasion of breast cancer cells including TNBC.

\section{Materials and Methods}

Human breast cancer cell lines. The normal breast epithelial cells MCF10A and the breast cancer cell lines MDA-MB-231 (basal type, TNBC), MCF-7 (luminal-A type, estrogen receptor-positive breast cancer), SKBR3 (HER2-positive breast cancer), T47D (luminal-A type, estrogen receptor-positive breast cancer), BT474 (estrogen receptor-positive breast cancer), and BT20 (TNBC) were purchased from the American Type Culture Collection (Manassas, VA, USA) (6, 
7). MCF10A was maintained in Dulbecco's modified Eagle's medium (DMEM)/F-12 (1:1) medium (Lonza, Walkersville, MD, USA) supplemented with $10 \%$ fetal bovine serum (FBS; Gibco, Grand Island, NY, USA), $10 \mathrm{ng} / \mathrm{ml}$ epidermal growth factor, $0.5 \mu \mathrm{g} / \mathrm{ml}$ hydrocortisone, $100 \mathrm{ng} / \mathrm{ml}$ cholera toxin, and $10 \mu \mathrm{g} / \mathrm{ml}$ insulin. MDAMB-231, MCF7, and SK-BR3 were maintained in DMEM (Gibco) supplemented with $10 \%$ FBS. T-47D, BT-20, and BT-474 cells were cultured in RPMI (Gibco) supplemented with 10\% FBS.

RNA extraction and lncRNA profiling. Total RNA was isolated from cells using RNAiso Plus (Takara, Otsu, Japan), in accordance with the manufacturer's instructions. RNA concentration was measured using NanoDrop ND-2000 (Thermo Scientific, Wilmington, DE, USA). RNA was treated with DNA-free ${ }^{\mathrm{TM}}$ DNase Treatment and Removal Reagents (Life Technologies, Carlsbad, CA, USA). For lncRNA expression profiling, $2 \mu \mathrm{g}$ of total RNA was reversetranscribed to cDNA using Human LncProfilers ${ }^{\mathrm{TM}}$ qPCR Array Kits (System Biosciences, Mountain View, CA, USA), in accordance with the manufacturer's recommendations.

Confirmation by quantitative reverse transcription-polymerase chain reaction ( $R T-P C R)$. In order to confirm the LncProfilers ${ }^{\mathrm{TM}}$ qPCR Array results, the total RNA in breast cancer cells was extracted using RNAiso Plus (Takara) and was treated with DNAfree $^{\text {TM }}$ DNase Treatment and Removal Reagents (Life Technologies). Reverse transcription was performed using Superscript III RT (Invitrogen, Carlsbad, CA, USA) and random hexamer (ELPIS-Biotech Inc., Daejeon, South Korea), following the manufacturer's protocol. Real-time PCR was performed for relative quantification of the expression levels of each lncRNA using Power SYBR Green PCR Master Mix (Applied Biosystems, Foster City, CA, USA). The $2^{-\Delta \Delta C T}$ method was used to determine relative gene expression, and the level of expression was normalized to that of $\beta$ actin $(A C T B)$. The primer sequences for real-time PCR were as follows: snaR: 5'-ATT GTG GCT CAG GCC GGT T-3' and 5'-TTT TTC CGA CCC ATG TGG ACC-3'; urothelial cancer associated 1 (UCA1): 5'-CCC AAG GAA CAT CTC ACC AA-3' and 5'-GAT GGT CCA AGG GGC TTC-3'; antisense noncoding RNA in the INK4 locus (ANRIL): 5'-TTG TTA GAA ACC AGG CTG CAC-3' and 5'-TTC TCT CTT TCT GTG GTT TCT CAA T-3; 200nucleotide-long non-messenger RNA (BC200): 5'-GCC CAG GAG TTC GAG ACC-3' and 5'-GCT TTG AGG GAA GTT ACG CTT AT-3'; small nucleolar RNA host gene 5 (SNHG5): 5'-GAG CAG CTC TGA AGA TGC AA-3' and 5'-TTT TAA CCA AGC GAT TTT CCA-3'; ACTB: 5'-TTG CCG ACA GGA TGC AGA A-3' and 5'GCC GAT CCA CAC GGA GTA CT-3'.

siRNA transfection. siRNAs specific to snaR and control RNA were synthesized by Cosmogentech (Seoul, South Korea). The sequence of si-snaR was 5'-CCA CAU GGG UCG GAA AAA AUU-3', and the sequence for the negative control (NC), not showing homology with the human genome, was 5'-UUC UCC GAA CGU GUC ACG UTT-3' (12).

Cells were inoculated in a six-well plate $\left(2 \times 10^{5} /\right.$ well $)$ for the wound-healing assay or a 96 -well plate $\left(5 \times 10^{3} /\right.$ well $)$ for the $3-(4,5-$ dimethylthiazol-2yl)-2,5-diphenyltetrazolium bromide (MTT) assay. After culture overnight, cells were transfected using Lipofectamine RNAiMAX reagent (Invitrogen) with the synthesized specific snaR or control siRNA. At $48 \mathrm{~h}$ after transfection, total RNA was extracted and the silencing effect was determined by real-time RT-PCR.
MTT assay. The effect of siRNA interference for snaR on the proliferation of breast cancer cells was determined by the MTT assay (Sigma, St. Louis, MO, USA). Cells at a total volume of $200 \mu$ were inoculated in a 96 -well plate, with $5 \times 10^{3}$ cells in each well. At each time point, $50 \mu \mathrm{l}$ of MTT $(2 \mathrm{mg} / \mathrm{ml})$ was added to the wells and the cells were cultured at $37^{\circ} \mathrm{C}$ for $4 \mathrm{~h}$. After removal of the medium, $100 \mu \mathrm{l}$ of dimethyl sulfoxide was added and the mixture was shaken for $10 \mathrm{~min}$. The optical density was then determined at $570 \mathrm{~nm}$. All of the experiments were conducted in triplicate.

Wound-healing assay. In order to determine effect of siRNA interference for snaR on cell migration, MDA-MB-231 cells were seeded in six-well culture plates and transfected with negative control siRNA or si-snaR. Next, a line was scratched into the cell monolayer using a sterile pipette tip, and the cells were further incubated. Images were then captured with the aid of a microscope after $0,24,48 \mathrm{~h}$ of incubation. The data are representative of three independent experiments.

Cell invasion assay. For the invasion assay, transwell chambers with $8-\mu \mathrm{m}$ pores were coated with Matrigel (Corning Inc., Tewksbury, MA, USA) and incubated at $37^{\circ} \mathrm{C}$ for $2 \mathrm{~h}$, allowing it to solidify. After transfection, cells were resuspended in DMEM containing $1 \%$ FBS and plated in the upper chamber at a density of $1 \times 10^{4}$ cells. The lower chamber contained complete medium supplemented with $10 \%$ FBS. After incubation for $48 \mathrm{~h}$, the cells on the internal surface of the chamber bottom were wiped with a cotton swab, fixed with $2 \%$ paraformaldehyde, stained with $0.5 \%$ crystal violet, and rinsed with phosphate-buffered saline. Cells were counted in four random fields for each well chambers under a light microscope.

Statistical analysis. Data are presented as the mean \pm standard deviation (SD) of three or more independent experiments. The differences in experimental results between two groups were analyzed using Student's $t$-test and a statistically significant difference was defined at $p<0.05$.

\section{Results}

snaR was significantly up-regulated in breast cancer cell lines. We screened for lncRNAs associated with the breast cancer cells MDA-MB-231 and MCF7, which represent triple-negative breast cancer and estrogen receptor-positive breast cancer, respectively, using qPCR array analyses. Among 90 lncRNAs, we extracted transcripts that were upregulated (expression change $>2$-fold) or down-regulated (expression change $<0.5$-fold) relative to MCF10A and analyzed them. E2F transcription factor 4, p107/p130binding (E2F4) antisense, insulin-like growth factor 2 antisense (IGF2AS), snaR, and SNHG5 were shown to be upregulated in MDA-MB-231 cells and 7SK, ANRIL, IGF2AS, Nespas, p53 mRNA, and snaR were found to be up-regulated in MCF-7 cells. In addition, BC200, Scm-related gene containing four mbt domains (SFMBT2), maternally expressed 9 (MEG9), nucleotide transport (NTT), UCA1, and yeast ribonucleic acid 1 (YRNAI) were down-regulated in MDA-MB-231, and SFMBT2 and YRNAI were downregulated in MCF-7 (Tables I and II). 
Confirmation of transcript expression by real-time RT-PCR in various breast cancer cell lines. In order to confirm the validity of the array methods, five of the 15 transcripts that showed significant up- or down-regulation in the array analysis were selected and subjected to real-time RT-PCR using RNAs from breast cancer cell lines MCF-10A (normal breast epithelial cells), MDA-MB-231 (basal type, TNBC), MCF-7 (luminal-A type, estrogen receptor-positive breast cancer), SKBR3 (HER2-positive breast cancer), T47D (luminal-A type, estrogen receptor-positive breast cancer), BT474 (estrogen-receptor-positive breast cancer), and BT20 (TNBC). The expression of each lncRNA in the breast cancer cell lines is shown in Figure 1. The expression of snaR, ANRIL, and SNHG5 in the breast cancer cell lines was higher than in normal breast cells. In particular, the expression of snaR was significantly high in the breast cancer cell lines compared with that in normal breast cells. Finally, $U C A 1$ and $B C 200$ were significantly down-regulated in the breast cancer cell lines.

Knockdown of snaR inhibits proliferation, migration, and invasion of $M D A-M B-231$ cells. Because snaR was found to be significantly up-regulated in MDA-MB-231 and MCF7 cells, we selected it from among those dysregulated in breast cancer cell lines. In order to investigate the functional role of snaR in breast cancer cells, we treated the MDA-MB-231 cells with an siRNA that knocks down snaR. We also determined the efficiency of this interference by quantitative RT-PCR. The specific siRNA was shown to induce significant down-regulation of the expression of snaR in MDA-MB-231 cells (Figure 2A). Upon this down-regulation of snaR, the proliferation of MDA-MB-231 cells was significantly inhibited, as determined by the MTT assay (Figure 2B). Furthermore, the migration of MDA-MB-231 cells was found to decrease upon this treatment in a woundhealing assay (Figure 2C). Functionally, down-regulation of snaR by siRNA was also undertaken in a third experiment to assess the invasiveness of MDA-MB-231 cells in an invasion assay. This showed that the invasiveness of MDA-MB-231 cells was also significantly reduced by the down-regulation of snaR compared to that in the NC cells (Figure 3). These combined results (proliferation, migration, and invasiveness) for the down-regulation of snaR in MDA-MB-231 cells show that this lncRNA is strongly associated with tumor progression.

\section{Discussion}

LncRNAs, which are a major part of the noncoding RNA family, are important modulators and regulators in various malignancies. Numerous studies on various types of lncRNA have demonstrated their diverse regulation of biological function and the knockdown of specific
Table I. Details of long noncoding RNA expression levels in MDA-MB231 breast cancer cell line.

\begin{tabular}{lcc}
\hline $\begin{array}{l}\text { Long noncoding } \\
\text { RNA }\end{array}$ & $\begin{array}{c}\text { NCBI reference } \\
\text { sequence }\end{array}$ & $\begin{array}{c}\text { Expression in MDA-MB-231 } \\
\text { relative to MCF10A cells }\end{array}$ \\
\hline snaR & NR_004435.1 & $9.60 \pm 1.50$ \\
E2F4 antisense & N/A & $3.41 \pm 1.63$ \\
SNHG5 & NR_003038.2 & $2.01 \pm 0.17$ \\
$I G F 2 A S$ (family) & N/A & $1.99 \pm 0.56$ \\
$N T T$ & U54776.1 & $0.57 \pm 0.12$ \\
$Y R N A-1$ & NR_004391.1 & $0.54 \pm 0.03$ \\
$M E G 9$ & EU616813.1 & $0.38 \pm 0.10$ \\
$U C A 1$ & NR_015379.3 & $0.26 \pm 0.11$ \\
$B C 200$ & AF020057.2 & $0.16 \pm 0.02$ \\
SFMBT2 & N/A & $0.02 \pm 0.03$ \\
\end{tabular}

NCBI: National Center for Biotechnology Information; N/A; NCBI reference sequence information not available.

Table II. Details of the long noncoding RNA expression levels in the MCF7 breast cancer cell line.

\begin{tabular}{lcc}
\hline $\begin{array}{l}\text { Long noncoding } \\
\text { RNA }\end{array}$ & $\begin{array}{c}\text { NCBI reference } \\
\text { sequence }\end{array}$ & $\begin{array}{c}\text { Expression in MCF7 } \\
\text { relative to MCF10A cells }\end{array}$ \\
\hline ANRIL & DQ485453.1 & $57.04 \pm 16.62$ \\
snaR & NR_004435.1 & $16.82 \pm 3.44$ \\
7 SK & N/A & $3.53 \pm 0.46$ \\
IGF2AS (family) & N/A & $3.28 \pm 0.33$ \\
Nespas & AJ251759.1 & $1.93 \pm 0.36$ \\
p53 mRNA & DA932053.1 & $1.81 \pm 0.51$ \\
DLG2AS (family) & N/A & $0.43 \pm 0.25$ \\
YRNA1 & NR_004391.1 & $0.35 \pm 0.04$ \\
SFMBT2 & N/A & $0.08 \pm 0.01$ \\
\hline
\end{tabular}

NCBI: National Center for Biotechnology Information; N/A; NCBI reference sequence information not available.

lncRNAs can have a reverse biological function, which can be applied to the treatment of cancer. For example, taurineup-regulated gene 1 (TUG1), an IncRNA, has been identified as being up-regulated in hepatocellular carcinoma; in addition, ANRIL, another lncRNA, is particularly up-regulated in familial melanoma bladder cancer (8-10). Furthermore, urothelial carcinoma-associated 1 (UCA1) has been identified as an oncogenic gene in nonsmall-cell lung cancer (11). Dysregulation of a specific lncRNA can thus play a biological role in promoting or inhibiting cell growth, proliferation, migration, or apoptosis. However, only few studies on lncRNAs in breast cancer have been performed (12-15).

In the current study, diverse breast cancer cell lines based on molecular subtype, namely MDA-MB-231, MCF7, SK- 
A snaR

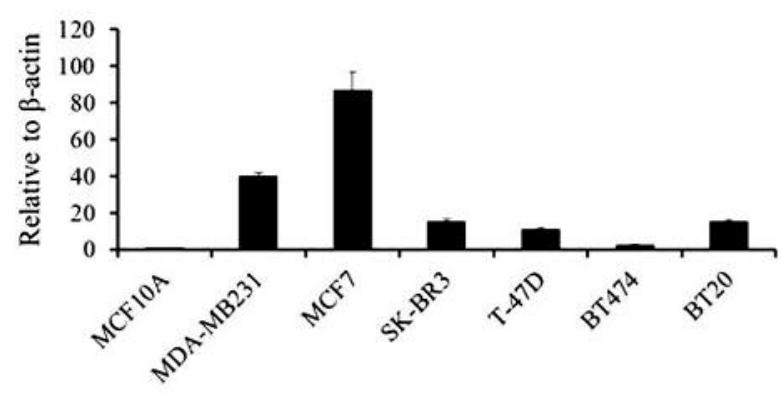

C

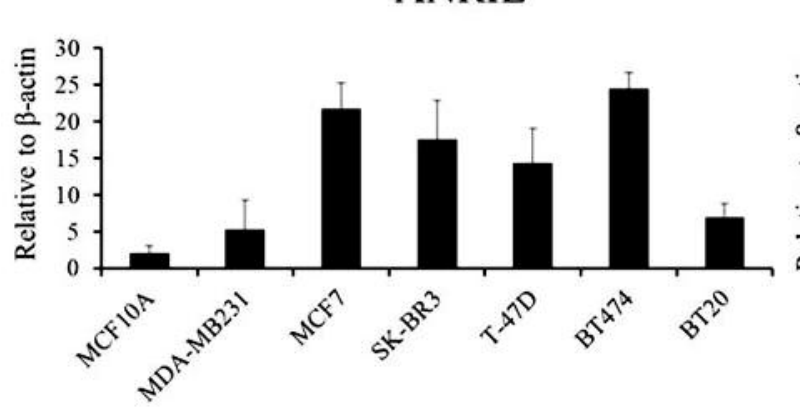

B

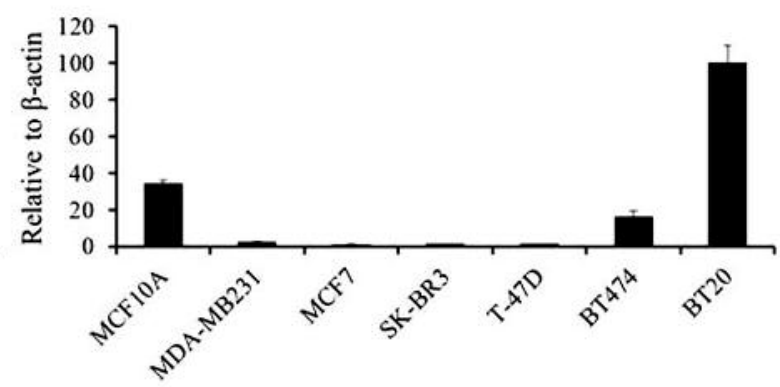

D

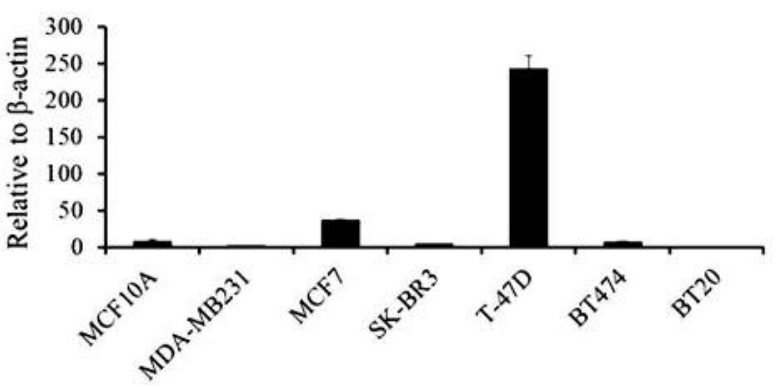

E

SNHG5

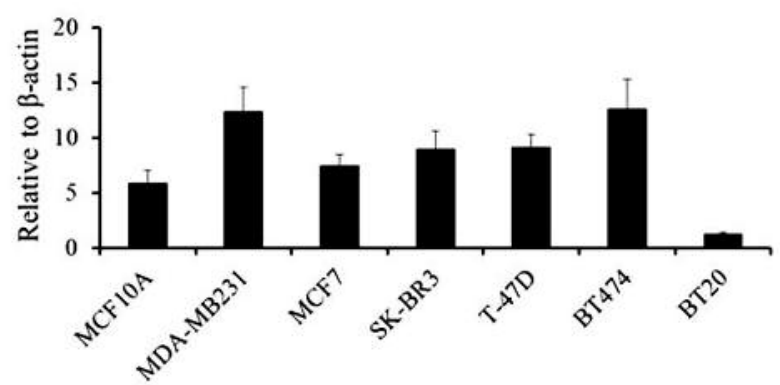

Figure 1. Confirmation study of the selected long noncoding RNA in different breast cancer cell lines. Small NF90-associated RNA (snaR) (A), urothelial cancer associated 1 (UCA1) (B), antisense noncoding RNA in the INK4 locus (ANRIL) (C), 200-nucleotide-long non-messenger RNA $(B C 200)(D)$, and small nucleolar RNA host gene 5 (SNHG5) (E), which were significantly up- or down-regulated in MDA-MB-231 or MCF7 cells, were selected, and quantitative reverse transcriptase polymerase chain reaction was performed for relative quantification of their expression levels in MCF10A, MDA-MB-231, MCF7, SK-BR3, T-47D, BT474, and BT20 cells. Data are presented as mean $\pm S D$ from three independent experiments.

BR3, T47D, BT474 and BT20, were used to explore the role of lncRNAs. Although various lncRNAs were expressed highly in each cell line, snaR and ANRIL were identified as being predominantly up-regulated in the TNBC cell line (MDA-MB-231) as well as the hormone receptor-expressing cell line (MCF7). In particular, snaR was shown to be 16.82 \pm 3.44 -fold (mean \pm SD) more highly expressed in the TNBC cell line.
snaR is a double-stranded RNA that binds important proteins implicated in multiple cellular functions by in vivo cross-linking followed by immunoprecipitation, indicating the possibility that it has a regulatory role in cancer development and progression $(16,17)$. Recently, snaR was, for example, identified to play a role in chemosensitivity to 5-fluorouracil in colon cancer cell lines, suggesting its potential usefulness for cancer treatment (18). 
A

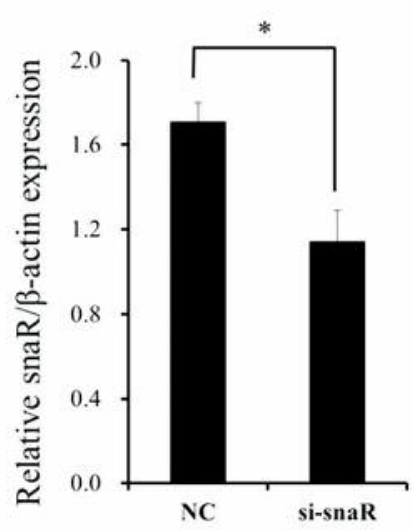

B

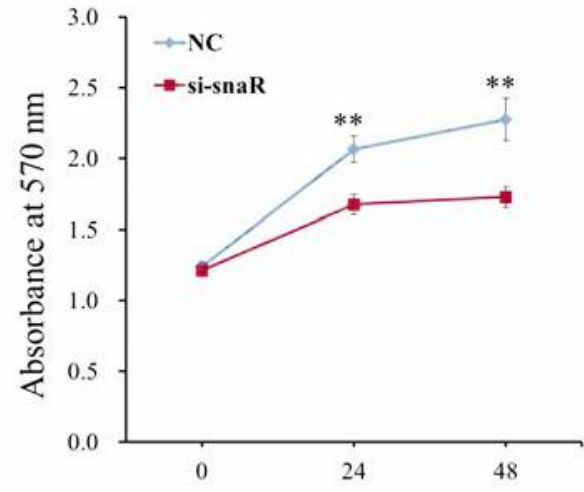

Culture time (h)
C
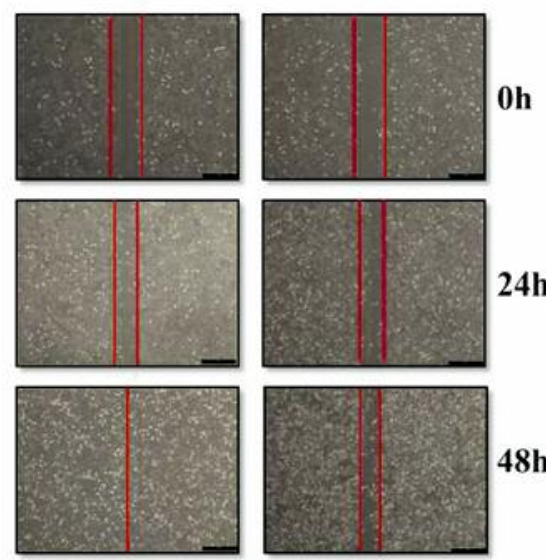

NC

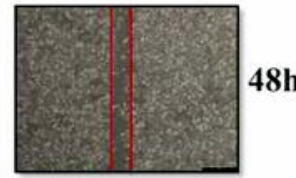

si-snaR

Figure 2. Knockdown of small NF90-associated RNA (snaR) suppresses the proliferation and migration of MDA-MB-231 cells. A: The level of snaR expression was determined in MDA-MB-231 cells upon transfection with si-snaR or negative control scramble (NC) by quantitative reverse transcriptase polymerase chain reaction. B: Cell proliferation assay following transfection with si-snaR or NC. C: Wound-healing assay. MDAMB-231 cells were transfected, cultured to monolayer and scratched with a pipette tip, and images were taken at 0, 24, and 48 h after wound formation. Data are presented as mean $\pm S D$. Significantly different at $* p<0.05$, and $* * p<0.005$ compared with the NC group.

A
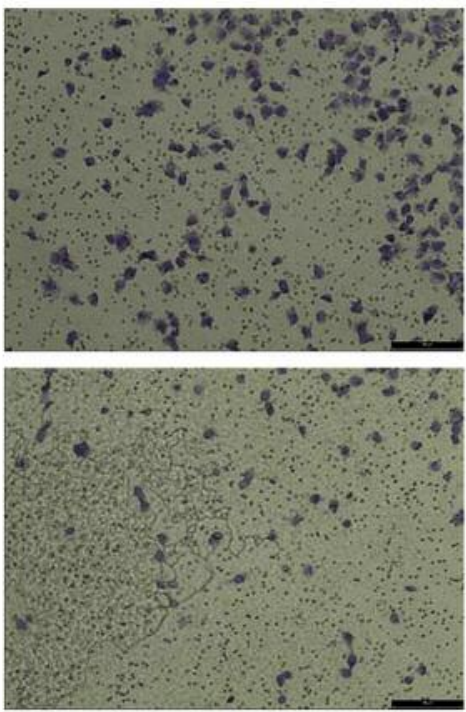

B

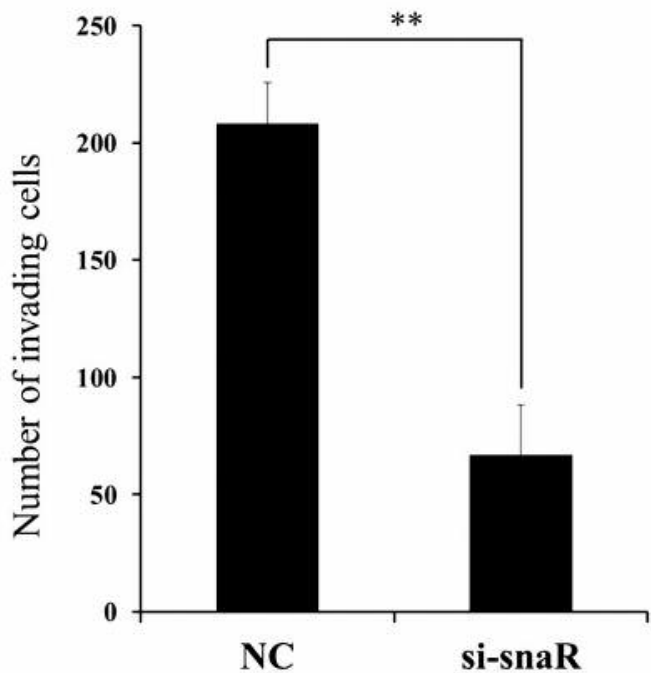

Figure 3. The effects of small NF90-associated RNA (snaR) knockdown on MDA-MB-231 cell invasion. A: The cell invasion analysis of MDA-MB231 cells upon transfection with si-snaR or negative control scramble $(N C)$ was performed by transwell assays with Matrigel-coated membrane. B: The number of invasive cells per field is shown. Data are presented as mean $\pm S D$. **Significantly different at $p<0.005$ compared with the NC group.

Recent evidence has also revealed that ANRIL regulates the proliferation, apoptosis, and metastasis of cancer cells in hepatocellular carcinoma, and lung, ovarian and gastric cancer, but not breast cancer (19-25). Although the expression of ANRIL in breast cancer cell lines was up- regulated, this lncRNA was also expressed in normal breast cells. In contrast, the difference in expression of snaR was more significant in breast cancer cell lines than in ANRIL when compared to the normal breast cell line. The functional study with the knockdown of snaR proved that blockage of 
biological function in snaR improves antitumoral efficacy by inhibiting cancer cell proliferation, migration and invasion.

TNBC confers poorer outcomes than other types of breast cancer (26). Because this group of breast cancer is characterized by a lack of expression of genes encoding hormone receptors as well as HER2, any hormone treatment or target should improve the outcome of patients with this disease. This explains the difficulty of finding an effective treatment for TNBC besides chemotherapy. However, based on several studies, several lncRNAs have been shown to be up-regulated or overexpressed specifically in TNBC (27, 28).

We assumed that a specific up-regulated lncRNA can easily be inhibited by a knockdown technique, although increasing the expression of a specific lncRNA that is down-regulated in breast cancer would be difficult. In the current study, snaR was found to be significantly upregulated in MDA-MB-231, TNBC, cells, and its expression was significantly inhibited by knockdown, as we expected. Consequently, the proliferation, migration, and invasion of breast cancer cells were significantly inhibited. If the knockdown of snaR can be applied clinically to TNBC, it would provide an innovative treatment for such cancer.

To the best of our knowledge, this is the first report of lncRNA snaR in breast cancer cell lines, showing that it regulates the proliferation, migration and invasion of cancer cells. However, there are still several barriers to applying a knockdown technique in treating breast cancer. Firstly, a further confirmatory study should be conducted at the tissue level in breast cancer, as well as an in vivo study. Secondly, we have yet to clarify the mechanism by which snaR significantly inhibited the proliferation, migration and invasion of TNBC cells. Further studies with breast cancer tissue should supply more information that could potentially lead to a novel treatment of breast cancer.

\section{Conclusion}

The IncRNA snaR was found to be up-regulated in breast cancer cell lines, especially in TNBC cells, and the proliferation, migration and invasion of TNBC cells were significantly suppressed by the knockdown of snaR. This study and further work may lead to snaR becoming a promising biomarker and therapeutic targeting agent for clinical application in TNBC.

\section{Acknowledgements}

This study was supported by a grant from the National R\&D Program for Cancer Control, Ministry of Health and Welfare, Sejong, Republic of Korea (1420040) and the National Research Foundation of Korea (NRF) grant funded by the Korean government (2014R1A5A2009242).

\section{References}

1 Perez DS, Hoage TR, Pritchett JR, Ducharme-Smith AL, Halling ML, Ganapathiraju SC, Streng PS and Smith DI: Long, abundantly expressed non-coding transcripts are altered in cancer. Hum Mol Genet 17: 642-655, 2008.

2 Wang KC and Chang HY: Molecular mechanisms of long noncoding RNAs. Mol Cell 43: 904-914, 2011.

3 Hauptman $\mathrm{N}$ and Glavač D: Long non-coding RNA in cancer. Int J Mol Sci 14: 4655-4669, 2013.

4 Serghiou S, Kyriakopoulou A and Ioannidis JP: Long noncoding RNAs as novel predictors of survival in human cancer: a systematic review and meta-analysis. Mol Cancer 15: 50, 2016.

5 Parrott AM and Mathews MB: Novel rapidly evolving hominid RNAs bind nuclear factor 90 and display tissue-restricted distribution. Nucleic Acids Res 35: 6249-6258, 2007.

6 Holliday DL and Speirs V: Choosing the right cell line for breast cancer research. Breast Cancer Res 13: 215, 2011.

7 van Slooten HJ, Bonsing BA, Hiller AJ, Colbern GT, van Dierendonck JH, Cornelisse CJ and Smith HS: Outgrowth of BT-474 human breast cancer cells in immune-deficient mice: a new in vivo model for hormone-dependent breast cancer. Br J Cancer 72: 22-30, 1995.

8 Rivenbark AG, O'Connor SM and Coleman WB: Molecular and cellular heterogeneity in breast cancer: challenges for personalized medicine. Am J Pathol 183: 1113-1124, 2013.

9 Huang MD, Chen WM, Qi FZ, Sun M, Xu TP, Ma P and Shu YQ: Long non-coding RNA TUG1 is up-regulated in hepatocellular carcinoma and promotes cell growth and apoptosis by epigenetically silencing of KLF2. Mol Cancer 14: 165, 2015.

10 Pasmant E, Laurendeau I, Héron D, Vidaud M, Vidaud D and Bièche I: Characterization of a germ-line deletion, including the entire INK4/ARF locus, in a melanoma-neural system tumor family: identification of $A N R I L$, an antisense noncoding RNA whose expression coclusters with $A R F$. Cancer Res 67: 39633969, 2007.

11 Zhu H, Li X, Song Y, Zhang P, Xiao Y and Xing Y: Long noncoding RNA ANRIL is up-regulated in bladder cancer and regulates bladder cancer cell proliferation and apoptosis through the intrinsic pathway. Biochem Biophys Res Commun 467: 223$228,2015$.

12 Nie W, Ge HJ, Yang XQ, Sun X, Huang H, Tao X, Chen WS and Li B: LncRNA-UCA1 exerts oncogenic functions in non-small cell lung cancer by targeting miR-193a-3p. Cancer Lett 371: 99106, 2016.

13 Mendell JT: Targeting a long noncoding RNA in breast cancer. N Engl J Med 374: 2287-2289, 2016.

14 Liu Y, Sharma S and Watabe K: Roles of lncRNA in breast cancer. Front Biosci 7: 94-108, 2015.

15 Milevskiy MJ, Al-Ejeh F, Saunus JM, Northwood KS, Bailey PJ, Betts JA, McCart Reed AE, Nephew KP, Stone A, Gee JM, Dowhan DH, Dray E, Shewan AM, French JD, Edwards SL, Clark SJ, Lakhani SR and Brown MA: Long-range regulators of the IncRNA HOTAIR enhance its prognostic potential in breast cancer. Hum Mol Genet 4: 177, 2016. [Epub ahead of print]

16 Reichman TW, Muñiz LC and Mathews MB: The RNA-binding protein nuclear factor 90 functions as both a positive and negative regulator of gene expression in mammalian cells. Mol Cell Biol 22: 343-356, 2002. 
17 Guo W, Wang Q, Zhan Y, Chen X, Yu Q, Zhang J, Wang Y, Xu $\mathrm{XJ}$ and $\mathrm{Zhu} \mathrm{L}$ : Transcriptome sequencing uncovers a three-long noncoding RNA signature in predicting breast cancer survival. Sci Rep 6: 27931, 2016.

18 Lee H, Kim C, Ku JL, Kim W, Yoon SK, Kuh HJ, Lee JH, Nam SW and Lee EK: A long non-coding RNA snaR contributes to 5-fluorouracil resistance in human colon cancer cells. Mol Cells 37: 540-546, 2014.

19 Kang YH, Kim D and Jin EJ: Down-regulation of phospholipase D stimulates death of lung cancer cells involving up-regulation of the long ncRNA ANRIL. Anticancer Res 35: 2795-2803, 2015.

20 Huang MD, Chen WM, Qi FZ, Xia R, Sun M, Xu TP, Yin L, Zhang EB, De W and Shu YQ: Long non-coding RNA ANRIL is up-regulated in hepatocellular carcinoma and regulates cell apoptosis by epigenetic silencing of KLF2. J Hematol Oncol 8: 50, 2015.

21 Huang MD, Chen WM, Qi FZ, Xia R, Sun M, Xu TP, Yin L, Zhang EB, De W and Shu YQ: Long non-coding RNA ANRIL is up-regulated in hepatocellular carcinoma and regulates cell proliferation by epigenetic silencing of KLF2. J Hematol Oncol 8: 57, 2015.

22 Qiu JJ, Lin YY, Ding JX, Feng WW, Jin HY and Hua KQ: Long non-coding RNA ANRIL predicts poor prognosis and promotes invasion/metastasis in serous ovarian cancer. Int $\mathrm{J}$ Oncol 46: 2497-2505, 2015.

23 Hua L, Wang CY, Yao KH, Chen JT, Zhang JJ and Ma WL: High expression of long non-coding RNA ANRIL is associated with poor prognosis in hepatocellular carcinoma. Int J Clin Exp Pathol 8: 3076-3082, 2015.
24 Nie FQ, Sun M, Yang JS, Xie M, Xu TP, Xia R, Liu YW, Liu $\mathrm{XH}$, Zhang EB, Lu KH and Shu YQ: Long noncoding RNA $A N R I L$ promotes non-small cell lung cancer cell proliferation and inhibits apoptosis by silencing $K L F 2$ and $P 21$ expression. Mol Cancer Ther 14: 268-277, 2015.

25 Lin L, Gu ZT, Chen WH and Cao KJ: Increased expression of the long non-coding RNA ANRIL promotes lung cancer cell metastasis and correlates with poor prognosis. Diagn Pathol 10: 14, 2015.

26 Ovcaricek T, Frkovic SG, Matos E, Mozina B and Borstnar S: Triple-negative breast cancer - prognostic factors and survival. Radiol Oncol 45: 46-52, 2011.

27 Yang F, Liu YH, Dong SY, Yao ZH, Lv L, Ma RM, Dai XX, Wang J, Zhang XH and Wang OC: Co-expression networks revealed potential core lncRNAs in the triple-negative breast cancer. Gene 591: 471-477, 2016.

28 Shen X, Xie B, Ma Z, Yu W, Wang W, Xu D, Yan X, Chen B, Yu L, Li J, Chen X, Ding K and Cao F: Identification of novel long non-coding RNAs in triple-negative breast cancer. Oncotarget 6: 21730-21739, 2015.
Received September 28, 2016

Revised October 17, 2016

Accepted October 20, 2016 